\title{
Wie Carl-Bertil Laurell und Sten Eriksson vor 50 Jahren den Alpha-1-Antitrypsin- Mangel entdeckten und was danach kam - ein etwas eigenwilliger persönlicher Rückblick
}

\author{
How Carl-Bertil Laurell and Sten Eriksson Detected the Alpha-1-Antitrypsin Deficiency 50 Years Ago and \\ What Then Came - A Somewhat Headstrong and Personal Retrospection
}

\section{Bibliografie \\ DOI http://dx.doi.org/ \\ 10.1055/s-0033-1344745 \\ Pneumologie 2013; 67: 562-566 \\ (c) Georg Thieme Verlag KG \\ Stuttgart · New York \\ ISSN 0934-8387}

\section{Korrespondenzadresse \\ Prof. Dr. med.}

Nikolaus Konietzko

Spillheide 78

45239 Essen

nikolaus.konietzko@t-online.de

\section{Die Entdeckung des Alpha-1-Antitrypsin- Mangels \\ $\checkmark$}

Vor 5000 Jahren muss es sich wohl ereignet haben. Damals herrschte noch Eiszeit und Europa lag unter einem dicken Eispanzer. Irgendwo in Skandinavien, so vermuten Epidemiologen, muss es zur Spontanmutation gekommen sein: Das normale, für die Produktion des Alpha-1-Antitrypsins verantwortliche M-Allel mutierte zum pathologischen Z-Allel. Das Z-Allel, etwas salopp auch als „Wikinger-Allel“ bezeichnet, ist die wichtigste Gen-Variante, die zum Alpha-1-Antitrypsin-Mangel führt. Von Skandinavien aus breitete sich die Mutation sukzessive nach Süden aus. Noch heute lässt sich - auch innerhalb Deutschlands - ein Nord-Süd-Gefälle bei der Z-Mutante nachweisen [1] . Dabei waren die damaligen Klimabedingungen für seine Verbreitung durchaus nicht hinderlich, wie man auf den ersten Blick vermuten könnte, sondern eher förderlich. Denn der Gendefekt - autosomal rezessiv vererbt, wie wir heute wissen - konnte im dünn besiedelten Norden Europas mit stark beschränkter Partnerwahl, möglicherweise auch Inzucht, leichter und „unverdünnter“ von einer Generation auf die andere weitergegeben werden als in dicht besiedelten Regionen - ein in der Genetik wohl bekanntes Phänomen.

Logischerweise fand die Geschichte des Alpha-1Antitrypsin-Mangels ihre Fortsetzung in Schweden. Hier an der Universität Lund hatte dessen Laborleiter Carl-Bertil Laurell die Technik der Elektrophorese, die er bei seinem Lehrer, dem Nobelpreisträger Arne Tiselius erlernt hatte, perfektioniert ( $\bullet$ Abb.1). Laurell war Anfang der 1960er-Jahre bereits ein berühmter Mann, hatte diverse biologisch wichtige Proteine wie Transferrin, Caeruloplasmin, Haptoglobin entdeckt und deren Funktion aufgeklärt. Auch hatte er mit dem schwedischen Hämatolgen Jan Gösta Waldenström, der Tür an Tür mit ihm arbeitete, das
„M“'-Globulin bei der Waldenströmschen Makroglobulinämie gefunden [2]. Als Leiter des Labors der Universität Lund ließ er jede Blutprobe, derer er habhaft werden konnte, mit „seiner“ Elektrophorese untersuchen. Jeden Papierausdruck, der dabei entstand, überprüfte er persönlich.

Eines Tages - es war im Herbst 1962 - kam ihm ein Streifen mit einem fehlenden Alpha-1-Globulin in die Hand, kurz darauf ein zweiter $(\bullet$ Abb.2). Beide stammten von Patienten aus dem nahe gelegenen „Malmö General Hospital“ der Universität Lund. Sten Eriksson, damals 30 Jahre alt, arbeitete dort als Assistenzarzt. Laurell rief ihn an und bat ihn, in seinem Labor in Lund vorbeizukommen. Er habe ihm etwas zu zeigen. Rasch erkannten beide - der Laborarzt Laurell und der Kliniker Eriksson - die Bedeutung ihrer Entdeckung und begannen mit einer systematischen Studie. Gemeinsam überprüften sie 1500 nicht selektionierte Elektrophorese-Streifen aus dem Laborfundus. Bei vier weiteren Fällen wurden sie fündig, sie hatten die nämliche Konstellation. Von den nunmehr sechs Patienten mit diesem Muster, also der fehlenden Alpha-1-Globulin-Fraktion, litten vier an einem Lungenemphysem.

Wenig später veröffentlichten Laurell und Eriksson ihre Beobachtungen im Scandinavian Journal of Clinical \& Laboratory Investigation. Sie vermieden es in Anbetracht der kleinen Fallzahl, weitgehende Schlussfolgerungen zu ziehen, äußerten aber die Vermutung, dass es sich um einen genetischen Defekt handeln könnte und dass die fehlende Alpha-1-Globulin-Fraktion augenfällig mit einem Lungenemphysem in Verbindung zu bringen sei. Sie gaben aber auch zu bedenken, dass die Lungenfunktion nicht in jedem Fall mit fehlender Alpha-1-Globulin-Fraktion beeinträchtigt sei [3]. Rasch war klar, dass sich hinter der Alpha-1-Globulin-Fraktion das Alpha-1-Antitrypsin verbarg, dessen Struktur und Funktion bereits 

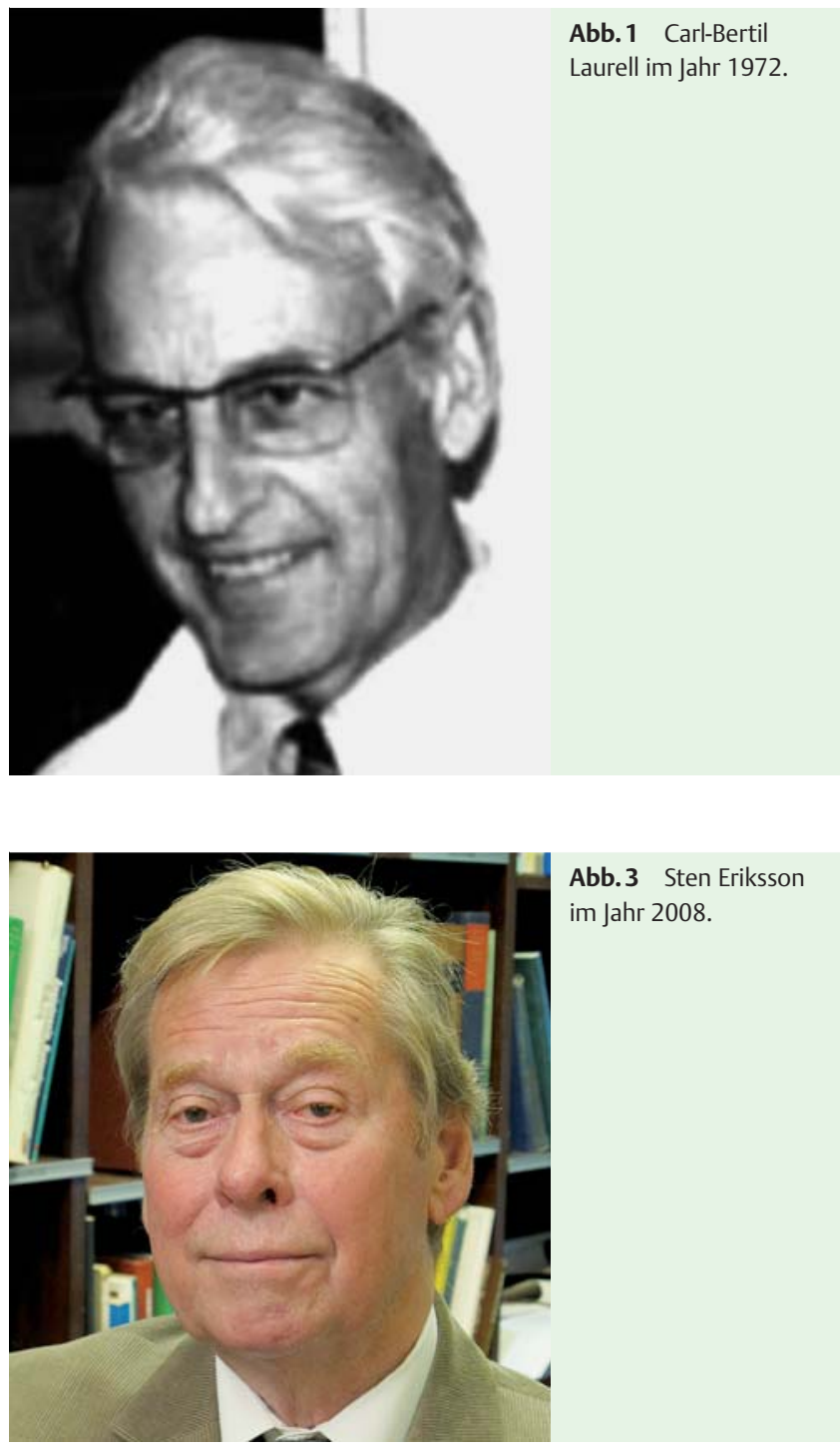

Abb. 3 Sten Eriksson im Jahr 2008

ein Jahr zuvor von Marburger Forschern aufgeklärt worden war [4]. Damit wurde 1963, das Jahr, in dem die Studie publiziert wurde, zum Geburtsjahr der Entdeckung des Alpha-1-Antitrypsin-Mangels, wenngleich die eigentliche Entdeckung ein Jahr zuvor erfolgt war.

Die Beobachtung der beiden Schweden faszinierte die Wissenschaftler in aller Welt und stimulierte eine Reihe von Studien in den unterschiedlichsten Disziplinen. Eriksson selbst ( $\bullet$ Abb.3) fand im folgenden Jahr weitere 33 Patienten und Familienmitglieder mit einem schweren Alpha-1-Antitrypsin-Mangel und konnte 1965 in seiner PhD-Arbeit die genetische Basis des Defekts sichern [5]. Carrell und Travis erforschten die Systematik der Serpine [6] und Fagerhol gelang die Phänotypisierung der verschiedenen Genvarianten, insbesondere der klinisch bedeutsamen S und Z-Varianten [7]. Er perfektionierte die Methode so, dass sie lange Zeit - auch vor Gericht - als Vaterschaftsnachweis genutzt wurde. 1970 konnte Harvey Sharp den Zusammenhang zwischen Leberzirrhose und Alpha-1-Antitrypsin-Mangel beweisen, später auch das gehäufte Vorkommen von hepato-zellulärem Leberkarzinom [8].

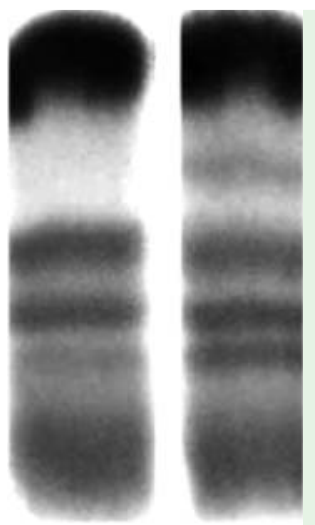

Abb.2 Fehlende Alpha-1-Bande im oberen Drittel des linken Streifens (Original aus [3]).

\section{Die These von der Proteasen-Antiproteasen-Imbalance $\nabla$}

1964 gelang es dem deutschstämmigen Pathologen Paul Gross mittels Papain, einer pflanzlichen Protease, bei der Ratte ein Lungenemphysem zu erzeugen [9]. Ich selbst schlug mich in den Jahren 1967/68 während eines Fellowships an der University of Illinois in Chicago mit einem Tiermodell zur Erzeugung eines Lungenemphysems herum. Dabei wurde die explantierte Lunge von Hamstern mit in Kochsalz gelösten Proteasen durchströmt und die dynamische Compliance kontinuierlich mitgemessen. Die Wirkung war durchschlagend, doch leider wurde nicht nur Lungengewebe zerstört, sondern auch die viszerale Pleura. Es entstanden multiple Lecks, sodass eine Messung der Compliance nicht mehr möglich war. Damit schien für mich das Kapitel „Alpha-1-Antitrypsin-Mangel“ beendet.

Im selben Jahr entdeckte Aaron Janoff in menschlichen Granulozyten das Enzym Elastase und mutmaßte, dass es Lungengewebe zerstören würde und somit als Verursacher des Lungenemphysems infrage käme. Später konnte er zeigen, dass das Alpha-1Antitrypsin-Molekül die Wirkung der neutrophilen Elastase blockiert und dass der protektive Effekt von Alpha-1-Antitrypsin durch Zigarettenrauch verloren geht [10]. Aus all diesen Bausteinen wurde dann peu à peu die Theorie von der Proteasen-Antiproteasen-Imbalance als Ursache für die Entstehung des Lungenemphysems zusammengepuzzelt ( $\bullet$ Abb.4).

Diese Theorie besagt, dass normalerweise in der Lunge eine Balance zwischen Proteasen und Antiproteasen besteht. Die wichtigste Protease ist die aus den Granulozyten stammende neutrophile Elastase, die wichtigste Antiprotease das intakte Alpha-1Antitrypsin. Fehlt letzteres, wie beim angeborenen Alpha-1-Antitrypsin-Mangel, überwiegen die destruktiven Kräfte der Proteasen, es kommt zur Zerstörung von Lungengewebe, zum Lungenemphysem. Ähnlich könnte die Pathogenese bei dem viel häufigeren Lungenemphysem des Zigarettenrauchers ablaufen: Da durch den Zigarettenrauch das Alpha-1-Antitrypsin funktionell unwirksam wird und als Antiprotease entfällt, entsteht quasi ein erworbener Alpha-1-Antitrypsin-Mangel mit den entsprechenden Konsequenzen.

\section{Vom Labor zum Krankenbett und wieder zurück $\nabla$}

10 Jahre später - ich war aus den USA zurückgekehrt und auf dem Umweg über die Universität Ulm, wo ich mich habilitiert hatte, in Essen gelandet - konsultierte mich ein knapp 40-jähriger Mann wegen zunehmender Atemnot bei körperlicher Belastung. Von 

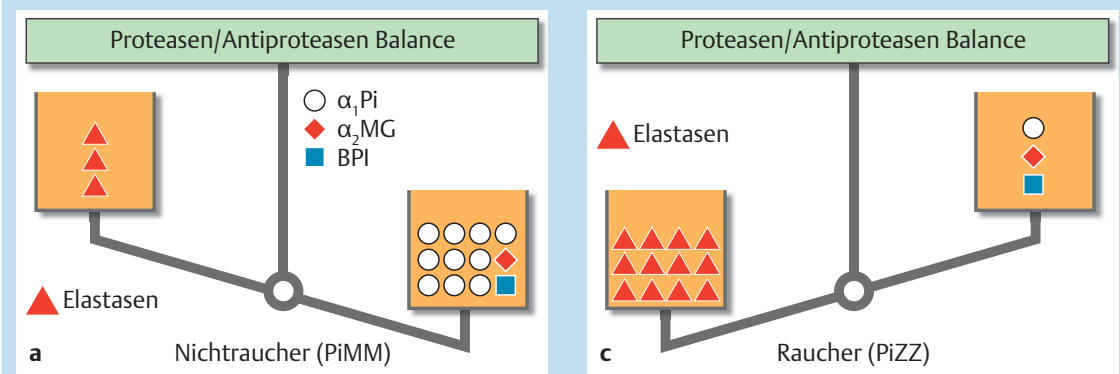

Abb.4 Proteasen-Antiproteasen-Balance bei verschiedenen Phänotypen $(\mathrm{PiMM}=$ normal, PiZZ = Alpha-1-AT-Mangel) und unterschiedlichem Raucherstatus sowie unter Substitution mit Alpha-1-AT-Konzentrat. a Nichtraucher b Raucher c Raucher mit Alpha-1-ATMangel d Patient mit Alpha-1-AT-Mangel unter Substitution. $\alpha_{1} \mathrm{Pi}=\alpha_{1}$-Proteaseninhibitor $\alpha_{2} \mathrm{MG}=\alpha_{2}-$ Makroglobulin $\mathrm{BPI}=$ bronchusprotektiver Inhibitor
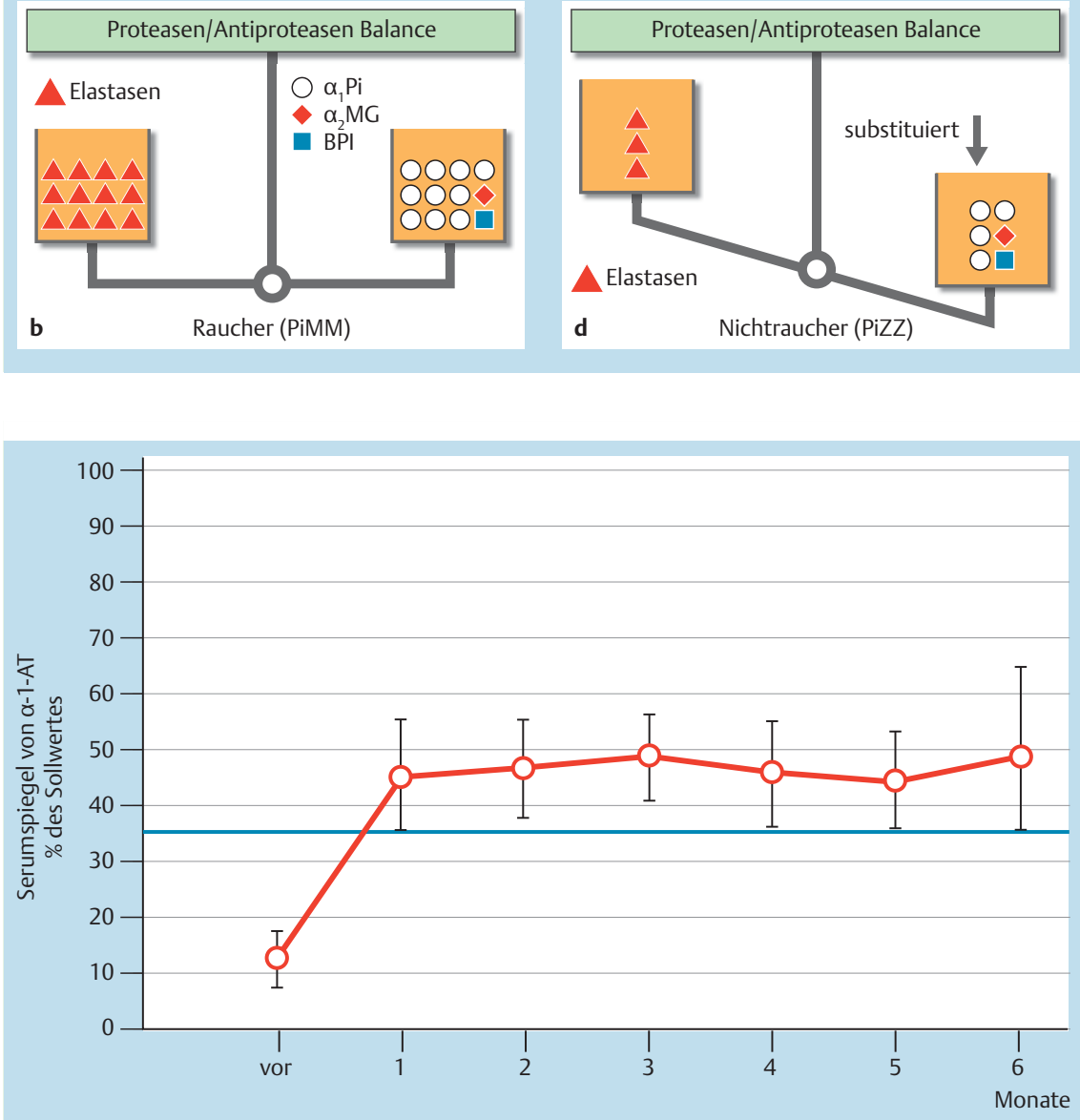

Abb.5 Tal-Spiegel von Alpha-1-AT im Serum bei 12 Patienten mit schwerem Alpha-1-AT-Mangel unter wöchentlicher Substitutionstherapie.

Beruf war er Kameramann beim ZDF. Er habe immer größere Schwierigkeiten, mit seiner (damals noch mehrere Kilogramm schweren) Kamera auf gleicher Höhe mit davoneilenden Politkern zu bleiben, etwa wenn er den ausgreifenden Schritten des späteren Bundeskanzlers Kohl zu folgen versuche. Bei der klinischen Untersuchung fielen mir lediglich diskrete Rasselgeräusche über beiden Lungenbasen auf ${ }^{1}$, der übrige Befund war regelrecht, ebenso wie das Röntgenbild des Thorax und die Ganzkörper-Plethysmografie. Differenzialdiagnostisch dachte ich an eine beginnende, Röntgen-invisible Lungenfibrose und bestimmte deswegen die Lungen-Compliance. Zu meiner Überraschung war diese erhöht, es lag also nicht, wie erwartet, eine „steife Lunge“ vor, sondern eine „schlaffe Lunge“ - Leitsymptom eines Lungenem-

\footnotetext{
${ }^{1}$ Dieses Auskultationsphänomen ist mir auch später immer wieder begegnet, ich habe es allerdings nie systematisch untersucht. Ich vermute, dass das Rasselgeräusch verursacht wird durch Bronchiektasen, die bekanntlich beim Alpha-1-Antitrypsin-Mangel nicht selten auftreten.
}

physems. Der Nachweis eines stark erniedrigten Alpha-1-Antitrypsins im Blut bestätigte die Diagnose: Der Patient litt offensichtlich an einem Lungenemphysem. Mein erster „Alpha-Patient".

Nachdem ich Herrn Denker, so hieß der Patient, die Diagnose eröffnet und mit ihm die Konsequenzen der Erkrankung eingehend besprochen hatte, begann er sich intensiv mit seiner Krankheit zu befassen, es war seine Art, sich mit seinem Schicksal auseinanderzusetzen. Ich bestärkte ihn darin. Er las alles, was zu dem Thema publiziert wurde, und stellte immer wieder bohrende Fragen. Damit forderte er uns Ärzte und trieb uns immer wieder an. Über 12 Jahre protokollierte er seine Krankheitssymptome und bestimmte täglich seinen Atemstoß (FEV1), veröffentlichte diese einmalige Kasuistik auch in „Respiration“ [11]. Deswegen kann ich hier seinen Namen nennen. Er war der erste Patient in Europa, dem das kommerzielle Alpha-1-Antitrypsin-Konzentrat infundiert wurde, und einer der ersten, die doppelseitig lungentransplantiert wurden. Nach der Transplantation hatte er noch fünf gute Jahre. Dann machte ihm eine chronische Abstoßungs- 
reaktion der Lunge mehr und mehr zu schaffen und er verstarb mit 57 Jahren.

Zurück ins Labor: In den späten 1970er-Jahren beschäftigte sich eine Forschergruppe am NIH in Bethesda, zu denen auch Claus Vogelmeier zählte, unter Führung von Ron Crystal intensiv mit diesem Thema, tierexperimentell und klinisch. Sie bestimmten die Konzentration sowohl der Elastase als auch des Antitrypsins in der BAL, zuerst bei Gesunden, dann bei Alpha-1-AntitrypsinDefizienten. Mit Hilfe dieser Daten errechneten sie einen „minimal protective level", der in der Lunge erreicht werden musste, und die benötigte Menge Alpha-1-Antitrypsin-Konzentrats, die man dem Mangelträger mindestens infundieren musste. Das gelang, die Patienten vertrugen die Infusion gut und der Schutzschild erwies sich über eine Woche lang als effektiv [12], Damit war natürlich nur bewiesen, dass es möglich ist, das Protease-Antiproteasen-Gleichgewicht durch Gabe von Alpha-1-Antitrypsin wieder herzustellen, nicht aber der Nachweis erbracht, dass sich damit auch die Entstehung und das Fortschreiten des Lungenemphysems verhindern ließ.

\section{Die Etablierung des Alpha-1-Antitrypsin-Registers in Deutschland}

Die Pharmaindustrie hatte unterdessen nicht geschlafen. Bayer war zu dieser Zeit über seine US-amerikanische Firma Cutter führend im Plasmageschäft, vor allem bei der Gewinnung von Gerinnungsfaktoren und Immunglobulinen. Was lag also näher, als die Plasmafraktion mit dem Alpha-1-Antitrypsin, die man bis dato „in den Ausguss geschüttet“ hatte, aufzuarbeiten und zu vermarkten. Das Hauptproblem dabei war die Sterilität, das Konzentrat musste frei von HI- und Hepatitis-Viren sein. Und das im gerade beginnenden AIDS-Zeitalter, als noch kein HIV-Test zur Verfügung stand. Die Chemiker von Cutter schafften es schlussendlich mit verschiedenen Tricks, ein steriles Präparat herzustellen, was im Nachhinein auch hunderttausendfach bewiesen werden konnte: Es wurde in all den folgenden Jahren über keine einzige Infektion mit den gefürchteten Viren berichtet.

Während also die Chemiker an der Produktion eines sicheren Alpha-1-Antitrypsin-Konzentrats aus humanem Plasma arbeiteten, stellten sich die Kliniker die Frage: Wo sind denn nun die Patienten mit dem schweren Alpha-1-Antitrypsin-Mangel, die möglicherweise für eine Dauersubstitution infrage kommen? Allein für das damalige Westdeutschland war deren Zahl aufgrund von Blutbank-Untersuchungen auf 6000 bis 8000 geschätzt worden. Schon bald wurden zwei Dinge klar:

1. Nur ein Bruchteil der Mangelträger war bisher identifiziert und

2. Wir wussten so gut wie nichts über den Verlauf der

Erkrankung.

Die logische Konsequenz dieser Erkenntnis war die Etablierung eines Patienten-Registers. Anfangs der 1980er-Jahre wurde innerhalb der „Wissenschaftlichen Arbeitsgemeinschaft zur Therapie von Lungenkrankheiten“, kurz WATL genannt, eine Arbeitsgruppe gegründet, die sich die Identifizierung, Dokumentation und Verlaufsbeobachtung von Patienten mit schwerem Alpha-1Antitrypsin-Mangel zum Ziel setzte. Mithilfe eines einseitigen Fragebogens wurden neben den biographischen Daten der klinische Status, die Lungenfunktion und der Alpha-1-AntitrypsinSpiegel im Serum abgefragt. Das Echo war unerwartet groß, und bis 1988 waren 488 Patienten mit einem Alpha-1-AntitrypsinSerumspiegel $\leq 35 \%$ vom Sollwert erfasst, auch Patienten aus
Österreich und der deutschsprachigen Schweiz. Damit besaß die WATL weltweit den größten Datenschatz zu dieser Thematik. Ein erstes, klinisch bedeutsames Ergebnis bei der Auswertung der Lungenfunktion war der quantifizierbare Nachweis der Progredienz des Lungenemphysems. Im Mittel zeigten Patienten mit schwerem Alpha-1-Antitrypsin-Mangel einen jährlichen Abfall des Atemstoßes von $120 \mathrm{ml}(\Delta \mathrm{FEV} 1 \geq 120 \mathrm{ml}$ ), wenn auch mit erheblichen interindividuellen Schwankungen. Zum Vergleich: Bei gesunden Nichtrauchern liegt der entsprechende Wert unter $20 \mathrm{ml}$ pro Jahr [13].

\section{Die bundesweite Implementierung der Substitutionstherapie \\ $\nabla$}

Die Zulassung des ersten humanen Alpha-1-Antitrypsin-Konzentrats, des Prolastin ${ }^{\circledR}$ der Firma Bayer, erfolgte 1989 in den USA und ein Jahr vorher in Deutschland, ohne dass kontrollierte, geschweige denn randomisierte Langzeitstudien vorlagen. Die Freigabe erfolgte aufgrund des biochemischen Nachweises der Wirksamkeit vor Ort, so wie sie vom NIH erbracht worden war, wurde aber von der Zulassungsbehörde mit der Auflage einer Anwendungsbeobachtung verbunden. Dank des bereits etablierten Patientenregisters der WATL konnte die breite Anwendung der Substitutionstherapie bundesweit zügig implementiert werden. Bis 1998 waren 443 Patienten mit Dauersubstitution in die Anwendungsbeobachtung einbezogen. Die Standarddosis musste in Einzelfällen angepasst werden, schwere Nebenwirkungen oder Infektionen durch das Blutprodukt traten nicht auf ( $\bullet$ Abb.5). Die halbjährigen Kontrollen unter Einschluss der Lungenfunktion wurden dezentral vorgenommen. Die Koordinierung der Studie erfolgte ebenso wie die Erfassung und Auswertung der Daten in Essen. Mit Hilfe des stattlichen Datenmaterials, das über die 12 Jahre seit der Zulassung von Prolastin ${ }^{\circledR}$ gesammelt worden war, konnte man sich an die Auswertung machen. Da eine Kontrollgruppe ohne Substitution in Deutschland nicht zur Verfügung stand Prolastin ${ }^{\circledR}$ war ja bereits zugelassen - kam die Idee auf, ein vergleichbares Kollektiv aus dem Dänischen Register heranzuziehen. Dank des Entgegenkommens unserer dänischen Kollegen konnte das Unterfangen in kurzer Zeit erfolgreich abgeschlossen werden. In dieser „Deutsch-Dänischen Verlaufsstudie“ wurde erstmals nachgewiesen, dass die Substitutionstherapie in der Lage ist, die Progredienz des Lungenemphysems bei schwerem Alpha-1-Antitrypsin-Mangel zu verlangsamen. Zumindest für die leichten bis mittelschweren Formen des Lungenemphysems (FEV1\%=31-65\% Soll) zeigte sich bei den dauersubstituierten deutschen Patienten im jährlichen FEV1-Abfall ein statistisch signifikanter Unterschied im Vergleich mit den nicht substituierten dänischen Patienten [14]. Die parallel laufende US-amerikanische „Alpha-1-Antitrypsin Deficiency Registry Study Group“ bestätigte die Ergebnisse ein Jahr später in den wesentlichen Punkten [15].

Die umfängliche, über zwei Dekaden fortgesetzte Datensammlung der „WATL“ ermöglichte es wenig später, auch eine Longitudinalstudie mit Serienmessungen der Lungenfunktion über mehrere Jahre durchzuführen. Wencker et al. konnten dabei zeigen, dass die Progredienz des Lungenemphysems bei PiZ-Mangel sich unter Substitution verlangsamt, besonders bei anfänglich foudroyantem Verlauf [16].

Seit den 1990er-Jahren lässt sich mit Hilfe der quantifizierten Computertomografie die Lungendichte messen. Für den Verlauf einer Erkrankung kann die Änderung der Lungendichte als Surro- 
gat-Parameter herangezogen und damit - in unserem Fall - die Progredienz des Lungenemphysems erfasst werden. Zwei Studien mit insgesamt 119 Patienten mit schwerem Alpha-1-Antitrypsin-Mangel liegen dazu vor und wurden kürzlich zusammenfassend ausgewertet. Dabei zeigte sich unter dauerhafter Substitution ein signifikant positiver Effekt hinsichtlich der Entwicklung der Gewebedichte [17]. Wir befinden uns also bei der Substitutionstherapie auf recht festem Boden, wenngleich nach wie vor Resultate kontrollierter randomisierter Studien fehlen.

\section{Ein Epilog}

Was hat Laurells und Erikssons Entdeckung heute vor 50 Jahren bewirkt? War sie bahnbrechend?

Ja, das war sie.

Wir Pneumologen haben mit der Entdeckung des Alpha-1-Antitrypsin-Mangels eine plausible Vorstellung von der Pathophysiologie des Lungenemphysems gewonnen - mit durchaus auch praktischen Konsequenzen für die alltägliche Praxis, insbesondere Prophylaxe und Therapie: Denken wir nur an die schädlichen Auswirkungen des Zigarettenrauchens, die beim angeborenen Alpha-1-Antitrypsin-Mangel besonders desaströs sind.

Viele unserer Patienten haben aus dem wissenschaftlichen Erkenntnisgewinn Nutzen gezogen. Die Substitutionstherapie ist deren augenfälligster Ausdruck: Über 4000 Patienten mit schwerem Alpha-1-Antitrypsin-Mangel erhalten gegenwärtig regelmäßige Infusionen mit Alpha-1-Antitrypsin-Konzentrat [18]. Neue Applikationstechniken wie die Inhalation von Alpha-1-Antitrypsin werden derzeit geprüft. Die Forschung auf diesem Gebiet läuft auf vollen Touren, Reparaturen am Gen oder Stammzelltherapie seien pars pro toto genannt.

Möglicherweise noch bedeutsamer als die oben beschriebenen wissenschaftlichen Fortschritte ist eine kürzlich gelungene Entdeckung: Seit einigen Jahren wissen wir, dass die Ursache des Alpha-1-Antitrypsin-Mangels auf einer Fehlfaltung in der Tertiärstruktur des Proteinmoleküls beruht. Mehr noch: Die Fehlfaltung des Moleküls ist nicht nur für die Unterfunktion des PiZAllels als Antiprotease und somit für die Entwicklung des Lungenemphysems verantwortlich, sondern auch für die Ablagerung des fehlgefalteten Proteins in den Leberzellen und damit die Entstehung der Leberzirrhose. Wir wissen heute aber auch, dass eine Reihe degenerativer Erkrankungen wie die Alzheimersche Erkrankung und der Morbus Parkinson diesem Formenkreis der „Confirmational diseases“ zuzurechnen ist [19]. Die Entdeckung des Alpha-1-Antitrypsin-Mangels durch Carl-Bertil Laurell und Sten Eriksson heute vor 50 Jahren könnte so den Grundstein gelegt haben für die Erforschung und vielleicht sogar Heilung der rätselhaftesten und unheimlichsten Krankheit unserer Zeit, der Alzheimerschen Erkrankung.

\section{Literatur}

1 Blanco I, de Serres FJ, Fernandez-Bustillo E et al. Estimated numbers and prevalence of $\mathrm{PI}^{*} \mathrm{PI}^{*} \mathrm{Z}$ alleles of $\alpha 1$-antitrypsin deficiency in European countries. Eur Respir J 2006; 27: 77-84

2 Carell $R W$. What We Owe to a1-Antitrypsin and to Carl-Bertil Laurell. COPD 2004; $1: 71-84$

3 Laurell C-B, Eriksson S. The electrophoretic a1- globulin pattern of serum in a1-antitrypsin deficiency. Scand J Clin Lab Invest 1963; 15: $132-140$

4 Schultze HE, Heide K, Haupt H. Alpha-1-antitrypsin aus Humanserum. Klin Wschr 1962; 40: 427-429

5 Eriksson S. Studies in a1-antitryspin deficiency. Acta Med Scand 1965; 432: $1-85$

6 Carrell $R$, Travis J. a1-antitrypsin and the serpins: variation and countervariation. Trends Biochem Sci 1985; 10: $20-24$

7 Fagerhol MK. The Pi system. Genetic variants of a1-antitrypsin. Ser Haematol 1968; 1: 153-161

8 Sharp HL, Bridges RA, Krivit W et al. Cirrhosis associated with alpha-1antitrypsin deficiency: a previously unrecognised inherited disorder. J Lab Clin Med 1969; 73: 934-939

9 Gross P et al. Experimental emphysema. Arch Environ Health 1965; 11: $50-58$

10 Janoff A, Carp H, Lee DK et al. Cigarette smoke inhalation decreases a1antitrypsin activity in rat lung. Science 1979; 206: 1313-1314

11 Wencker M, Denker J, Konietzko N. Serial measurement of FEV 1 over 12 years in a patient with alpha-1-protease inhibitor deficiency: Influence of augmentation therapy and infection. Respiration 1994; 61: $195-198$

12 Gadek JE, Klein HG, Holland PV et al. Replacement therapy of alpha-1antitrypsin deficiency: reversal of protease-antiprotease imbalance within alveolar structures of PiZ-subjects. J Clin Invest 1981; 68: $1158-1165$

13 Konietzko N, Schulz V, Eckert G. Die Progredienz des Lungenemphysems bei schwerem Alpha-1-PI-Mangel - eine retrospektive, multizentrische Studie der „Wissenschaftlichen Arbeitsgemeinschaft für die Therapie von Lungenkrankheiten (WATL)“. Med Klinik 1988; 83: $1-8$

14 Wencker M, Banik N, Viskum K et al. Does alpha1-antitrypsin augmentation therapy slow the annual decline in FEV1 in patients with severe hereditary alpha1-antitrypsin deficiency? Wissenschaftliche Arbeitsgemeinschaft zur Therapie von Lungenerkrankungen (WATL) alpha1AT study group Eur Respir J 1997; 10: 2260 - 3

15 The Alpha-1-Antitrypsin Deficiency Registry Study Group. Survival and FEV1 decline in individuals with severe deficiency of alpha1-antitrypsin. Am J Respir Crit Care Med 1998; 158: 49-59

16 Wencker M, Fuhrmann B, Banik $N$ et al. Longitudinal follow-up of patients with alpha-1-protease inhibitor deficiency before and during therapy with IV alpha-1-protease inhibitor. Chest 2001; 119: 737-44

17 Chapman KR, Stockley RA, Dawkins $C$ et al. Augmentation therapy for alpha1 antitrypsin deficiency: a meta-analysis. COPD 2009; 6: $177-$ 184

18 Köhnlein T, Welte T. Alpha1-antitrypsin deficiency: Pathogenesis, Clinical Presentation, Diagnosis, and Treatment. Am J Medicine 2008; 121: $1-9$

19 Carrell RW, Lomas DA. Alpha1-antitrypsin deficiency - a model for conformational diseases. N Engl J Med 2002; 346: 45-53 